\title{
Tratamiento actual de la enfermedad por reflujo gastroesofágico
}

\author{
Current treatment of gastroesophageal reflux disease \\ José de Jesús González-Izquierdo,* Tulio Jesús Hernández-Aguilar,* \\ Ulises Rodrigo Palomares-Chacón, ${ }^{* *}$ Luis Fernando Castelltor Hernández, ** \\ José Víctor Pérez Navarro, ${ }^{* *}$ Roberto Anaya-Prado**,***
}

Palabras clave: Enfermedad por reflujo gastroesofágico, funduplicatura, inhibidor de la bomba de protones.

Key words: Gastroesophageal reflux disease, fundoplication, proton pump inhibitor.

* Unidad de Atención Médica, Nivel Central, Instituto Mexicano del Seguro Social (IMSS), México DF, México.

** Departamento

de Cirugía General, Unidad Médica de Alta Especialidad Hospital de Especialidades (UMAEHE), Centro Médico Nacional de Occidente (CMNO) del IMSS, Guadalajara, Jalisco, México.

*** Dirección de Educación e Investigación en Salud del Hospital de Ginecología y Obstetricia, CMNO, IMSS.

\section{RESUMEN}

La enfermedad por reflujo gastroesofágico se define como una falla de la barrera antirreflujo que determina un paso anormal de secreciones digestivas altas al esófago y produce síntomas con daño esofágico y extraesofágico. Los mecanismos fisiológicos que impiden el reflujo son el aclaramiento, el esfínter esofágico inferior y el vaciamiento gástrico adecuado. El fracaso en estos mecanismos de defensa permite el reflujo gastroesofágico. El tratamiento quirúrgico de la enfermedad por reflujo gastroesofágico ha estado en constante evolución durante los últimos 70 años. La funduplicatura de Nissen, la de Belsey y la gastropexia de Hill han sido estudiadas y modificadas con el paso del tiempo; se han realizado diferentes tipos de abordajes. Un tratamiento efectivo es reducir la secreción ácida del estómago, bien sea con antagonistas de los receptores $\mathrm{H} 2$, bien con inhibidores de la bomba de protones; la dosis dependerá de la severidad de cada paciente, ya que cuanto mayor sea la exposición esofágica al ácido, mayor será el grado de supresión que se requerirá para sanar. Actualmente existe mucho debate en cuanto a los beneficios del tratamiento médico frente al quirúrgico y el papel de la laparoscopia como cirugía de primer tiempo o como segundo abordaje. El objetivo de este artículo es determinar las opciones terapéuticas quirúrgicas y médicas que tenemos actualmente en nuestro medio.

\section{ABSTRACT}

Gastroesophageal reflux disease is defined as a failure of the anti-reflux barrier that leads to abnormally high digestive secretions passing into the esophagus, which produces symptoms of esophageal and extra-esophageal damage. The physiological mechanisms that prevent reflux are clearance, the lower esophageal sphincter and the right gastric emptying. Failure in these defense mechanisms leads to gastroesophageal reflux. Surgical treatment of gastroesophageal reflux disease has been in constant evolution over the past 70 years. Nissen fundoplication, Belsey's and Hill gastropexy have been studied and modified over time, and with different approaches. An effective treatment is to reduce acid secretion of the stomach with either an $\mathrm{H} 2$ receptor antagonist or a proton pump inhibitor. The dose depends on the severity of each patient: the greater the esophageal exposure to acid, the greater will be the degree of suppression required to cure it. There is currently much debate about the benefits of the medical or surgical treatments, and the role of laparoscopic surgery as a first-time or second approach. The objective of this paper is to determine the surgical and medical treatment options currently available.

\section{INTRODUCCIÓN}

$\mathrm{E}_{\mathrm{p}}^{\mathrm{l}}$ tratamiento quirúrgico de la enfermedad por reflujo gastroesofágico (ERGE) ha estado en constante evolución durante los últimos 70 años. La funduplicatura tipo Nissen o tipo Belsey y la gastropexia de Hill han sido estudiadas y modificadas con el paso del tiempo; esto ha llevado a realizar diferentes tipos de abordajes, incluyendo la vía torácica o la abdo- minal; en épocas más recientes, se han utilizado los abordajes toracoscópicos, laparoscópicos y técnicas asistidas por robot.

En 1951 se creía que la hernia hiatal era la primera causante del reflujo. Siguiendo esta hipótesis, Allison publica ese mismo año su técnica para el tratamiento de la ERGE. A partir de esta hipótesis, en 1956, Rudolph Nissen publica su técnica en el Swiss Journal Schweizerische Medizine Wochenschrift, siendo esta técnica la 
más utilizada en la actualidad tras haber sufrido varias modificaciones: la primera en 1964 por Rossell y la segunda en 1985 por Donahue, introduciendo la funduplicatura flexible. En 1991 Dallemagne realiza la primera funduplicatura por vía laparoscópica. ${ }^{1}$

Actualmente existe mucho debate en cuanto a los beneficios del tratamiento médico frente al quirúrgico, el papel de la laparoscopia como cirugía de primer tiempo o como segundo abordaje, la necesidad de realizar alargamiento esofágico, funduplicaturas parciales $y$, recientemente, el uso de terapias endoluminales. Por lo tanto, estamos sumergidos en la evolución del tratamiento de la enfermedad por reflujo gastroesofágico (ERGE). ${ }^{1}$

\section{Epidemiología}

La ERGE se presenta en personas con una condición sintomática o alteración histopatológica resultante de episodios de reflujo gastroesofágico. ${ }^{1,2}$

En la población general, se ha reportado una prevalencia del $3-4 \%$ de esofagitis. Aunque la acidez es el síntoma más común de esofagitis, $70 \%$ de las personas con acidez intermitente no tienen esofagitis, en tanto que $60-95 \%$ de las personas con acidez diaria tienen esofagitis. ${ }^{2,3}$

Esta enfermedad tiene la misma prevalencia entre hombres y mujeres, pero hay una discreta preponderancia en el varón para esofagitis (2:1 versus $3: 1)$ y metaplasia de Barret $(10: 1)$. El embarazo se asocia con una mayor prevalencia de ERGE, pues $48-79 \%$ de las mujeres embarazadas se queja de acidez. Todas las formas de ERGE afectan más a la raza caucásica que a otras. $^{3-6}$

\section{Fisiopatología}

La anormalidad fundamental en el ERGE es la exposición del epitelio esofágico a la secreción gástrica, lo que resulta en una lesión histopatológica o en la presencia de síntomas. Pero cierto grado de reflujo gastroesofágico y exposición del epitelio al ácido se considera normal o fisiológico. La ERGE se presenta cuando la exposición del epitelio esofágico al jugo gástrico excede lo que el epitelio puede tolerar. Bajo condiciones normales, el reflujo de jugo gástri- co dentro del esófago distal se previene como una función de la unión gastroesofágica (UGE). La unión gastroesofágica es una zona anatómica cuya función es ser una barrera antirreflujo. Esta función se ha atribuido a múltiples mecanismos, aunque tienen consideración especial el esfínter esofágico inferior (EEI), la influencia del hiato diafragmático y la arquitectura muscular del cardias que constituye la parte distal (de alta presión) de la unión gastroesofágica. 7,8

Para mantener el delicado balance entre el flujo que pasa hacia el estómago y el que intenta regresar al esófago, el EEI tiene un complejo control neurológico que se relaciona con el sistema nervioso central y el sistema nervioso periférico. La presión del EEl es modulada por nervios vagales aferentes, así como simpáticos y vagales eferentes. La función eferente es mediada a través de neuronas del plexo mientérico que pueden producir contracción o relajación del EEl. ${ }^{8}$

El mecanismo protector contra el reflujo varía con las circunstancias fisiológicas. Esto es, el segmento intraabdominal del EEI es importante para prevenir el reflujo cuando se deglute y en reposo en decúbito; la crura diafragmática lo es cuando aumenta la presión intraabdominal. Cuando uno o más de estos mecanismos protectores fallan, el efecto deletéreo que sucede es aditivo, resultando en un aumento del número de eventos de reflujo $y$, consecuentemente, incrementando la exposición anormal del esófago al ácido. Las investigaciones se han enfocado en tres mecanismos principales de la incompetencia de la unión gastroesofágica: relajaciones transitorias del EEI sin anormalidad anatómica, hipotensión del EEI sin anormalidad anatómica, o deformación de la unión gastroesofágica, que incluye, aunque no se limita a, la hernia hiatal. El mecanismo que predomina depende de varios factores, que incluyen la anatomía de la unión gastroesofágica. ${ }^{8-11}$

\section{Diagnóstico}

Las herramientas con las que se cuenta actualmente para realizar el diagnóstico de ERGE son, de entrada, una historia clínica que incluya en la anamnesis la naturaleza de la sintomatología, como es la duración del episodio, síntomas 
nocturnos, si se presentan con la inclinación, paciente con estrés emocional, así como la aparición de la clínica con la ingesta de ciertos alimentos como café o, en su caso, tabaco. ${ }^{1-9}$

Los estudios de gabinete que se realizan para la aproximación diagnóstica incluyen, en primer lugar, un estudio baritado como el esofagograma, ya que éste representa la herramienta más útil para delimitar la anatomía del esófago, identificando su tamaño, así como la presencia o ausencia de hernia hiatal, datos importantes que ayudan a la selección del tipo de cirugía, además de que puede identificar estructuras que en la endoscopia no se pueden observar. ${ }^{12-15}$

El estudio endoscópico valora la mucosa esofágica; es útil para determinar la presencia de esofagitis y estimar el grado de severidad, además de otras enfermedades como divertículos, hernia hiatal, estenosis y tumoraciones, entre otras; pero su limitación está en la falla para estadificar el tamaño de la hernia, y no se debe realizar sólo con esta finalidad. ${ }^{12}$

La pHmetría es considerada el estándar de oro para valorar la presencia de reflujo. Se hace una medición cuantitativa de la exposición ácida al esófago cada 4-6 segundos; el paciente activa un marcador cuando siente los síntomas, está comiendo y con los cambios posturales. Se colocan los electrodos a cinco $\mathrm{cm}$ por arriba del EEl; otras pruebas los colocan en el estómago y $15 \mathrm{~cm}$ arriba del esfínter, teniendo que estar en promedio durante 24 horas. Con esta información se pueden calcular diferentes parámetros como el índice de DeMeester, que incluye porcentaje de tiempo total del $\mathrm{pH}<$ 4 , porcentaje de tiempo continuo de $\mathrm{pH}<4$, número total de episodios de reflujo, número de episodios de reflujo que duran más de cinco minutos. En individuos sin enfermedad, tiene una puntuación de 14 o menos, pero ésta es sólo una escala de referencia, ya que por sí sola no puede hacer diagnóstico de ERGE y regularmente se requieren estudios complementarios. ${ }^{13,14}$

La manometría, que es otro método de diagnóstico para el estudio del ERGE, brinda información en cuanto al funcionamiento del EEl y el cuerpo del esófago. Esto se traduce en detección de una motilidad esofágica anormal, como en la acalasia o esclerodermia; asimismo, permite identificar a los candidatos ideales para cirugía antirreflujo (deficiencia en el EEI y presión del esfínter $<6 \mathrm{mmHg}$, distancia menor a dos $\mathrm{cm}$ y porción intraabdominal menor a un $\mathrm{cm}$ ). Pacientes con ausencia de peristaltismo son candidatos a una funduplicatura parcial. ${ }^{13,14}$

\section{Tratamiento médico}

Un tratamiento efectivo de la ERGE es reducir la secreción ácida del estómago, bien con antagonistas de los receptores $\mathrm{H} 2$ o ya sea con inhibidores de la bomba de ácido; la dosis dependerá de la severidad de la enfermedad en cada paciente, ya que cuanto mayor sea la exposición esofágica al ácido, mayor será el grado de supresión que se requerirá para sanar. ${ }^{15-17}$

Muchos estudios se han realizado para establecer la eficacia de estos agentes en el tratamiento de la esofagitis. Los antagonistas de los receptores $\mathrm{H} 2$ han demostrado un efecto terapéutico del $10-24 \%$, en tanto que los inhibidores de la bomba de protones son más eficaces, con una mejoría del $57-74 \%$, en ambos casos en comparación con placebo. ${ }^{16-20}$

También se ha evaluado el tratamiento de la esofagitis severa resistente al manejo con los antagonistas de los receptores $\mathrm{H} 2$, con el uso de inhibidores de la bomba de ácido; se ha encontrado que la dosis de omeprazol a 40 $\mathrm{mg} /$ día mejora al $80 \%$ de estos pacientes dentro de un periodo de 12 semanas. Más aún, en un estudio holandés se encontró que el 100\% de los pacientes con esofagitis refractaria tuvo curación a las 20 semanas de tratamiento con omeprazol a esta dosis. ${ }^{21-26}$

Otra opción de tratamiento médico son los medicamentos procinéticos, que teóricamente aumentan la presión del EEI, mejorando el vaciamiento gástrico y la peristalsis. Estudios europeos que compararon la cisaprida a dosis de $10 \mathrm{mg}$ cuatro veces/día contra $150 \mathrm{mg}$ de ranitidina dos veces/día demostraron una eficacia similar para disminuir los síntomas de la ERGE y curar la esofagitis leve, pero ninguno tuvo algún efecto de mejoría en la esofagitis moderada o severa. En otro estudio donde se utilizó la cisaprida y la cimetidina, $70 \%$ de los pacientes tuvieron mejoría, en comparación con sólo $46 \%$ de los casos que recibieron cimetidina 
como monoterapia después de 6-12 semanas. Por lo tanto, los medicamentos procinéticos se pueden considerar como tratamientos de elección en las esofagitis leves. ${ }^{11,27,28}$

\section{Tratamiento quirúrgico}

Normalmente, la funduplicatura laparoscópica se ha considerado como el tratamiento de elección para la ERGE cuando falla el tratamiento médico de pacientes con esofagitis, sangrado de esofagitis refractaria o una hernia hiatal con erosiones transhiatales, síntomas extraesofágicos por la regurgitación, y en pacientes que prefieran la cirugía en lugar del manejo médico. ${ }^{1}$

Las indicaciones para el tratamiento quirúrgico son falta de respuesta al tratamiento médico y recurrencias frecuentes $y / 0$ complicaciones como el reflujo en posición supina, contractilidad deficiente del esófago, esofagitis erosiva, reflujo de contenido biliar y deficiencia estructural del esfínter. ${ }^{8,29,30}$

La cirugía antirreflujo es un tratamiento efectivo para el control del reflujo crónico. Desde el uso de la cirugía laparoscópica, dos de los procedimientos más comunes son la funduplicatura de Nissen y la de Toupet. Estos procedimientos reducen la hernia hiatal con la construcción de una válvula con el fondo gástri$\mathrm{Co}$, mecanismo que restablece la competencia de la unión gastroesofágica. Ésta ha demostrado índices de eficiencia de $90 \%$ cuando es practicada por cirujanos experimentados. ${ }^{1,31}$

Este tratamiento tiene un índice de conversión bajo de laparoscópico a abierto de 0-2.4\% en la literatura mundial. Las complicaciones intraoperatorias tampoco han sido elevadas, con un rango de $0-3.7 \%$ en diversos estudios, siendo la lesión esplénica, esofágica, hepática y pleural las más frecuentes. ${ }^{1,29-36}$

Las complicaciones postquirúrgicas tienen un rango muy amplio, dependiendo del estudio valorado, yendo desde $14 \%$ en el reportado por Anvari en 2006 hasta $0.9 \%$ del Reflux Trial en 2008. En otras series se describieron complicaciones de 3 y $5.5 \%$. Las principales causas de las complicaciones también fueron migración de la funduplicatura, estenosis esofágica e infección respiratoria. ${ }^{29-36}$

Existen principios de la cirugía como restaurar la presión del esfínter distal al doble de la porción en reposo $(12 \mathrm{mmHg})$, la longitud mínima que sea de $3 \mathrm{~cm}$, dejar un segmento intraabdominal mínimo de 1.5 a $2 \mathrm{~cm}$, lograr que el cardias reconstruido permita la deglución; además de que la funduplicatura no debe aumentar la resistencia del esfínter en la peristalsis esofágica; ésta tendrá que estar dentro del abdomen sin tensión y mantenerse en su sitio mediante la aproximación de los pilares del diafragma. ${ }^{8}$

Se advierte de un fallo en la cirugía cuando hay recurrencia o persistencia de los síntomas después de ésta, o incluso con la presencia de nuevos síntomas. El síntoma más común es la disfagia, a la que le sigue la saciedad temprana, distensión abdominal, diarrea y pirosis. El índice de reoperación es otro que varía según el autor, con rango de 0 a $3.7 \%{ }^{29-35} \mathrm{El}$ primer estudio a realizar es un esofagograma baritado, que en manos experimentadas identifica anormalidades anatómicas postquirúrgicas, las cuales se han clasificado en cuatro tipos, de: 1) rotura completa de la funduplicatura con recurrencia de la hernia hiatal, 2) parte del estómago deslizado por arriba del diafragma, 3) parte del estómago arriba de la funduplicatura pero debajo del diafragma, y 4) herniación de la funduplicatura sobre el hiato, estando dentro del tórax. ${ }^{37}$

\section{Tratamiento médico versus quirúrgico}

El debate en cuanto a cuál es el tratamiento ideal para la ERGE continúa, aunque la evidencia reciente se inclina en cierta medida por un manejo quirúrgico inicial que no todos los artículos avalan. ${ }^{36,38-47} \mathrm{Al}$ comparar ambas modalidades de tratamiento, el manejo médico se considera superior al quirúrgico respecto a la presencia de flatulencias, ya que estas aparecen con menor frecuencia en este grupo $(p<$ 0.001) de pacientes, así como el costo global, que en estos individuos es prácticamente un tercio en comparación con los que se someten a cirugía. ${ }^{38-47}$

La acidez y la disfagia fueron los síntomas que según la evidencia revisada no presentan diferencia en cuanto al tratamiento ofrecido (médico o quirúrgico). ${ }^{38-47}$ En donde se ha encontrado que la cirugía es superior al tratamiento médico es en la calidad de vida, pues 
se halló que los sujetos operados tuvieron una mejoría estadísticamente significativa a los tres meses y al año en comparación con los manejados médicamente; también el reflujo ácido reporta una mejoría mayor en el grupo quirúrgico, igual que la manometría. ${ }^{38-47}$

\section{CONCLUSIONES}

El costo del tratamiento quirúrgico es considerablemente superior al tratamiento médico; la funduplicatura laparoscópica brinda una mejoría en la calidad de vida y los síntomas relacionados con la ERGE en el corto y mediano plazo, aunque es incierto a largo plazo, principalmente en la acidez y el reflujo; también disminuye la exposición del esófago distal al ácido. Por lo tanto, con base en el grado de esofagitis de los pacientes y el conocimiento de los riesgos, costos y beneficios del tratamiento médico y quirúrgico para la ERGE, se deberá personalizar el tratamiento en cada caso. En todos los casos de ERGE es necesario dar oportunidad al tratamiento médico para mejorar la sintomatología; si ésta no mejora a pesar de un adecuado cumplimiento del tratamiento, se indica la opción quirúrgica, que genera resultados muy beneficiosos para los enfermos. En nuestro medio contamos con el recurso humano capacitado y la infraestructura necesaria para realizar procedimientos quirúrgicos para la ERGE como la funduplicatura completa o parcial con buenos resultados.

\section{CONFLICTO DE INTERESES}

Los autores declaran no tener ningún conflicto de intereses.

\section{REFERENCIAS}

1. Christian DJ, Buyske J. Current status of antireflux surgery. Surg Clin North Am. 2005; 85: 931-947.

2. Kahrilas P. Gastroesophageal reflux disease. JAMA. 1996; 276: 983-988.

3. Hirschowitz BI. Management of refractory and complicated reflux esophagitis. Yale J Biol Med. 1996; 69: 271-281.

4. El-Serag H, Hill C, Jones R. Systematic review: The epidemiology of gastro-oesophageal reflux disease in primary care, using the UK general practice research database. Aliment Pharmacol Ther. 2009; 5: 470-480.

5. Dent J, El-Serag HB, Wallander MA, et al. Epidemiology of gastro-oesophageal reflux disease: A systematic review. Gut. 2005; 54: 710-717.
6. Bainbridge ET, Temple JG, Nicholas SP, et al. Symptomatic gastro-oesophageal reflux in pregnancy: a comparative study of white Europeans and Asians in Birmingham. Br J Clin Pract. 1983; 37: 53-57.

7. Mittal R, Holloway R, Penagini R, et al. Transient lower esophageal sphincter relaxation. Gastroenterology. 1995; 109: 601-610.

8. Dent J, Dodds WJ, Friedman RH, et al. Mechanism of gastroesophageal reflux in recumbent asymptomatic human subjects. J Clin Invest. 1980;65:256-67.

9. Vakil N, van Zanten SV, Kahrilas PJ, et al., Global Consensus Group. The Montreal definition and classification of gastroesophageal reflux disease: a global evidence-based consensus. Am J Gastroenterol. 2006; 101: 1900-1920; quiz 1943.

10. Kahrilas PJ. Anatomy and physiology of the gastroesophageal function. Gastroenterol Clin North Am. 1997; 26: 467-486.

11. Richards WG, Sugarbaker DJ. Neuronal control of esophageal function. Chest Surg Clin N Am. 1995; 5: 157-171.

12. Lundell LR, Dent J, Bennett JR, et al. Endoscopic assessment of oesophagitis: clinical and functional correlates and further validation of the Los Angeles classification. Gut. 1999; 45: 172-180.

13. Zerbib F, Roman S, Ropert A, et al. Esophageal pHimpedance monitoring and symptom analysis in GERD: a study in patients off and on therapy. Am J Gastroenterol. 2006; 101: 1956-1963.

14. Hemmink GJ, Bredenoord AJ, Weusten BL, et al. Esophageal $\mathrm{pH}$-impedance monitoring in patients with therapy-resistant reflux symptoms: 'on' or 'off' proton pump inhibitor? Am J Gastroenterol. 2008; 103: 2446-2453.

15. Kitchin LI, Castell DO. Rationale and efficacy of conservative therapy for gastroesophageal reflux disease. Arch Intern Med.1991; 151: 448-454.

16. Bell N, Hunt R. Role of gastric acid suppression in the treatment of gastroesophageal reflux disease. Gut. 1992; 33: 118-124.

17. Harvey RF, Gordon PC, Hadley N, et al. Effects of sleeping with the bedhead raised and of ranitidine in patients with severe peptic oesophagitis. Lancet. 1987; 2: 1200-1203.

18. DeVault KR, Castell DO, American College of Gastroenterology. Updated guidelines for the diagnosis and treatment of gastroesophageal reflux disease. Am J Gastroenterol. 2005; 100: 190-200.

19. Mathias SD, Colwell HH, Miller DP, et al. Healthrelated quality-of-life and quality-days incrementally gained in symptomatic none rosive GERD patients treated with lansoprazole or ranitidine. Dig Dis Sci. 2001; 46: 2416-2423.

20. Sontag SJ. The medical management of reflux esophagitis. Role of antacids and acid inhibition. Gastroenterol Clin North Am. 1990; 19: 683-712.

21. Savarino V, Dulbecco P. Optimizing symptom relief and preventing complications in adults with gastrooesophageal reflux disease. Digestion. 2004; 69: 9-16.

22. Becher A, El-Serag H. Systematic review: the association between symptomatic response to proton pump inhibitors and health-related quality of life in patients with gastro-oesophageal reflux disease. Aliment Pharmacol Ther. 2011; 34: 618-627. 
23. Lind T, Havelund T, Carlsson R. Heartburn without esophagitis: efficacy of omeprazole therapy and features determining therapeutic response. Scand J Gastroenterol. 1997; 32: 974-979.

24. Hatlebakk J, Berstad A, Carling L, et al. Lansoprazole versus omeprazole in short-term treatment of reflux esophagitis: results of a Scandinavian multicentre trial. Scand J Gastroenterol. 1993; 28: 224-228.

25. Feldman M, Harford W, Fisher R, et al. Treatment of reflux esophagitis resistant to $\mathrm{H} 2$ receptor antagonists with lansoprazolea new $\mathrm{H}+/ \mathrm{K}$ sup + -ATPase inhibitor: a controlled, double-blind study. Am J Gastroenterol. 1993; 88: 1212-1217.

26. Klinkenberg-Knol E, Festen H, Jansen J, et al. Long term treatment with omeprazole for refractory reflux esophagitis: efficacy and safety. Ann Intern Med. 1994; 121: 161-167.

27. Richter J, Long J. Cisapride for gastroesophageal reflux disease: a placebo-controlled, double blind study. Am J Gastroenterol.1995; 90: 423-430.

28. Galmiche J, Brandsatter, Evreux M, et al. Combined therapy with cisapride and cimetidine in severe oesophagitis: a double blind controlled trial. Gut. 1988; 29: 675-681.

29. Catarci M, Gentileschi P, Papi C, et al. Evidence-based appraisal of anti-reflux fundoplication. Ann Surg. 2004; 239: 325-337.

30. Metz DC. Long-term outcome of laparoscopic Nissen and laparoscopic Toupet fundoplication for gastroesopahgeal reflux disease: a prospective, randomized trial. Surg Endosc. 2011; 24: 924-932.

31. Nijjar RS, Watson D, Jamieson GG, et al. Five-year follow-up of a multicenter, double-blind randomized clinical trial of laparoscopic Nissen vs anterior 90 degrees partial fundoplication. Arch Surg. 2010; 145: 552-557.

32. Markar SR, Karthikesalingam AP, Wagner OJ, et al. Systematic review and meta-analysis of laparoscopic Nissen fundoplication with or without division of the short gastric vessels. Br J Surg. 2011; 98: 1056-1062.

33. Koch OO, Kaindlstorfer A, Antoniou SA, et al. Laparoscopic Nissen versus Toupet fundoplication: objective and subjective results of a prospective randomized trial. Surg Endosc. 2012; 26: 413-422.

34. Oelschlager BK, Petersen RP, Brunt LM, et al. Laparoscopic paraesophageal hernia repair: Defining longterm clinical and anatomic outcomes. J Gastrointest Surg. 2012; 16: 453-459.

35. Attwood SEA, Lundell L, Ell C, et al. Standardization of surgical technique in antireflux surgery: the LOTUS trial experience. World J Surg. 2008; 32: 995-958.

36. Anvari M, Allen C, Marshall J, et al. A randomized controlled trial of laparoscopic Nissen fundoplication versus proton pump inhibitors for treatment of patients with chronic gastroesophageal reflux disease: one-year follow-up. Surg Innov. 2006; 13: 238-249.

37. Hunter JG, Smith CD, Branum GD, et al. Laparoscopic fundoplication failures: patterns of failure and response to fundoplication revision. Ann Surg. 1999; 230: 595604.
38. Willeman S, McCann S, Grant A, et al. Medical versus surgical management for gastro-oesophageal reflux disease (GORD) in adults. Cochrane Database Syst Rev. 2010; 17: CD003243.

39. Attwood SE, Lundell L, Hatlebakk JG, et al. Medical or surgical management of GERD patients with Barrett's esophagus: The LOTUS trial 3-year experience. J Gastrointest Surg. 2008; 12: 1646-1654.

40. Lundell L, Attwood S, Ell C, et al. Comparing laparoscopic antireflux surgery with esomeprazole in the management of patients with chronic gastrooesophageal reflux disease: a 3-year interim analysis of the LOTUS trial. Gut. 2008; 57: 1207-1213.

41. Cookson R, Flood C, Koo B, et al. Short-term cost effectiveness and long-term cost analysis comparing laparoscopic Nissen fundoplication with proton pump inhibitor maintenance for gastro-oesophageal reflux disease. Br J Surg. 2005; 92: 700-706.

42. Decadt B, Lowndes R, Rhodes M. Prospective randomized trial of laparoscopic Nissen fundoplication (LNF) versus maintenance proton pump inhibition (PPI) in the treatment of gastro-oesophageal reflux disease (GORD): manometric findings. Gastroenterology. 1999; 116: A146.

43. Grant A, Wileman S, Ramsay C, et al. The effectiveness and cost-effectiveness of minimal access surgery amongst people with gastro-oesophageal reflux disease -a UK collaborative study. The REFLUX trial. Health Technol Assess. 2008; 12: 1-181, iii-iv.

44. Grant AM, Wileman SM, Ramsay CL, et al. Minimal access surgery compared with medical management for chronic gastro-oesophageal reflux disease: UK collaborative randomised trial. BMJ. 2008; 337: a2664.

45. Metha S, Bennett J, Mahon D, et al. Prospective trial of laparoscopic Nissen fundoplication versus proton pump inhibitor therapy for gastroesophageal reflux disease: Seven-year follow-up. J Gastrointest Surg. 2006; 10: 1312-1317.

46. Anvari M, Allen C, Marshall J, et al. A randomized controlled trial of laparoscopic Nissen fundoplication versus proton pump inhibitors for the treatment of patients with chronic gastrooesophageal reflux disease (GERD): 3 year outcomes. Surg Endosc. 2011; 25: 2547-2554.

47. Faria R, Bojke L, Epstein D, et al. Cost effectiveness of laparoscopic fundoplication versus continued medical management for the treatment of GERD based on longterm follow-up of the REFLUX trial. Br J Surg. 2013; 100: 1205-1213.

Correspondencia:

Acad. Dr. en. C. Roberto Anaya Prado

Blvd. Puerta de Hierro 5150. Int 201B

Fraccionamiento Corporativo Zapopan.

Zapopan, Jal. 45110

Tel. y fax: (33) 3848 5410, (33) 38485411

Teléfono directo: (33) 34407691

E-mail: robana@prodigy.net.mx 